\title{
[1
}

TI 2019-040/I

Tinbergen Institute Discussion Paper

\section{Anticipating Peer Ranking Causes Hormonal Adaptations that Benefit Cognitive Performance}

Carsten de Dreu ${ }^{1}$

Klarita Gërxhani²

Arthur Schram ${ }^{3}$

\footnotetext{
${ }^{1}$ Leiden University

2 European University Institute

3 University of Amsterdam
} 
Tinbergen Institute is the graduate school and research institute in economics of Erasmus University Rotterdam, the University of Amsterdam and VU University Amsterdam.

Contact: discussionpapers@tinbergen.nl

More TI discussion papers can be downloaded at https://www.tinbergen.nl

Tinbergen Institute has two locations:

Tinbergen Institute Amsterdam

Gustav Mahlerplein 117

1082 MS Amsterdam

The Netherlands

Tel.: + 31(0)205984580

Tinbergen Institute Rotterdam

Burg. Oudlaan 50

3062 PA Rotterdam

The Netherlands

Tel.: +31(0)10408 8900 


\title{
Anticipating Peer Ranking Causes Hormonal Adaptations that Benefit Cognitive Performance
}

\author{
Carsten K.W. De Dreu ${ }^{1,2,},{ }^{*}$ Klarita Gërxhani ${ }^{3,{ }^{*}}$ \& Arthur Schram ${ }^{2,4,{ }^{*}}$
}

${ }^{1}$ Institute of Psychology, Leiden University (PB 9555, NL-2300 RB Leiden).

${ }^{2}$ Amsterdam School of Economics (PO Box 15867, NL-1001 NJ Amsterdam).

${ }^{3}$ Department of Political and Social Sciences, European University Institute, Florence.

${ }^{4}$ Robert Schumann Center for Advanced Studies, European University Institute, Florence.

${ }^{*}$ All authors contributed equally to this paper. Corresponding Author: C.K.W. De Dreu (c.k.w.de.dreu@fsw.leidenuniv.nl). 


\begin{abstract}
Performance ranking is common across a range of professional and recreational domains. Even when it has no economic consequences but does order people in terms of their social standing, anticipating such performance ranking may impact how people feel and perform. We examined this possibility by asking human subjects to execute a simple cognitive task while anticipating their performance being ranked by an outside evaluator. We measured baseline and post-performance levels of testosterone and cortisol. We find that (i) anticipating performance ranking reduces testosterone and increases cortisol; (ii) both these hormonal responses benefit cognitive performance; which explains why (iii) anticipation of being ranked by a peer increases cognitive performance.
\end{abstract}


People often want to know where they stand relative to others. They compete to estimate relative strength, curiously scrutinize the performance of others, and become upset when their social standing is undermined or otherwise below their expectations. Indeed, the drive to enhance and protect one's standing within groups and organizations is at the core of social cognition and behavior (Festinger, 1954; Jost, Banaj \& Nosek, 2004; Taylor \& Lobel, 1989). It is also seen in other social animals who show-off their strength, battle for high standing and suffer when falling behind (Twenge, et al., 2012; Sapolsky, 2005, 2017). In fact, in human groups and societies social comparisons are continuously and routinely made and are an intricate and institutionalized part of life. Whether at work, in the classroom, or when recreating, humans anticipate being compared and ranked for their performance.

While omnipresent and seemingly unavoidable, whether and how the anticipation of being ranked affects performance is poorly understood. In earlier work the anticipation of performance ranking invariably coincided with economic incentives, thus clouding interpretation (Anderson, Ertac, Gneezy, List \& Maximiano, 2013; Buser, Dreber, \& Mollerstrom, 2017; Buckert et al., 2015). Moreover, some studies suggest that anticipating performance ranking may deteriorate cognitive performance. For example, preparing for a competitive (versus cooperative) interaction enhances cognitive rigidity and reduces creative thinking (De Dreu \& Nijstad, 2008; Staw, Sandelands \& Dutton, 1981; Pashler, Johnson \& Ruthruff, 2001; Gneezy \& Rustichini, 2000; Chen, Williamson \& Zhou, 2012), and being observed by others increases stress reactions that slows down task performance (Kelsey, Blascovitch et al., 2000; Schmader, Johns, \& Forbes, 2008). Other studies suggest, in 
contrast, that anticipating performance ranking may improve cognitive performance (Schram, Brandts, \& Gerxhani, 2018). For example, relative to working alone, competitive incentives can be arousing (Harrison et al., 2001), and some level of socially-induced stress can promote cognitive performance (Oei et al., 2006). Accordingly, our first goal here was to examine whether the anticipation of being ranked influences cognitive performance in pure absence of economic incentives to outperform others.

Our second goal was to explore the possible mechanisms evoked by the anticipation of being ranked, focusing on adaptations in two key hormones - testosterone and cortisol. Testosterone belongs to the hypothalamic-pituitary-gonadal axis (HPG axis) and has a well-documented role in the regulation of social status and dominance-related behavior (Sapolsky, 2005; Cooke, Kavussanu, McIntyre \& Ring, 2014; Eisenegger \& Fehr, 2011; Newman, Sellers \& Joseph, 2005). In humans, testosterone associates with increased risktaking (Mazur \& Booth, 1998), more heuristic thought processes and reduced cognitive performance (Goucki \& Kimuram 1991). Cortisol belongs to the hypothalamic-pituitaryadrenal (HPA) axis. Cortisol has a well-documented role in stress-regulation (Sapolsky, 2005; Dickerson \& Kemeny, 2004). For example, stress-induced cortisol responses can augment cognitive control and analytical performance (Goldfarb et al., 2016). We predicted, accordingly, that (i) reductions in testosterone during task execution associate with increases in cognitive performance, and (ii) increases in cortisol during task execution associate with increases in cognitive performance. By implication, (iii) performanceenhancing effects of the anticipation of being ranked by a peer should be due to reduced levels of testosterone and increased levels of cortisol (Fig. 1BC). To examine these 
possibilities, we assessed changes in salivary testosterone and cortisol between the beginning of the experiment and upon completion of the cognitive performance task (and controlled for initial hormonal balance, gender, and lifestyle; Fig. 1BC).

\section{Materials and Method}

\section{Participants}

The experiment was run in June 2015 at the CREED laboratory of the University of Amsterdam. An earlier study on performance ranking and cognitive performance, using the same experimental set-up as used here, used sample sizes of 18 per treatment (Weber \& Schram, 2016). Using the results from that study we expected a medium effect size between $d=0.5$ (conservative) and $d=0.8$ (liberal) for the comparison between NR-T and PR-T. GPower 3.1 (Faul et al., 2007, with $\alpha=0.05$ and $1-\beta=0.80$, yielded a required sample size of 53 and 21 for each treatment, with an average of $N=37$. As these numbers were well above the sample sizes typically used in studies tracking neurohormonal responses in cognitive performance tasks, we determined our overall sample size for this study at $N=74$ and oversampled to rescue statistical power in case of equipment failure and outlier detection. In total, we recruited 95 participants, mainly undergraduate students at the University of Amsterdam. All of the more than 2000 potential participants in the CREED subject pool received an invitation to voluntary sign up and participation was on a first-come, first-serve basis. We organized eight sessions: four sessions with 13 and four with 18, for a total of 124 participants. 28 of these were passive C-players as described below and one participant (a type B-player, see below) decided to leave during the experiment. This leaves us with 95 participants for our data analysis. 


\section{Experimental Procedures and Treatments}

An outline of the experimental procedure is given in Fig 1A. At the beginning of a session, participants were told the rules of conduct. They were subsequently asked to open the closed envelop on their desk, which contained preliminary instructions. Participants read these privately, at their own pace. Instructions are reproduced in the SI. The experiment consists of two stages, as implemented in Schram et al. (2018). In Stage I (computerized), participants undertake an individual cognitive task. We call these 'active' participants. The experimental software was developed in Delphi at the Center for Research in Experimental Economics and political Decision making (CREED) by Jos Theelen. The software is available from the authors upon request. In Stage II (not computerized), some participants are required to report their result to a peer (i.e. non-active participant) seated in a separate office (Fig S1). Instructions for Stage II were distributed after Stage I was completed. Sessions lasted approximately 80 minutes, and participant earnings were performance based, with an average of €21.69 (including a $€ 7$ show-up fee) for active participants. Inactive participants received a flat fee of $€ 20$ (including a $€ 7$ show-up fee).

In each session, before entering the laboratory, each participant is randomly assigned to one of three player types, denoted by A, B and C. Only types A and B (active participants) enter the laboratory and do the cognitive task described below. C-players (non-active participants) are taken to separate rooms and act as peers. In every session there are six A-players and six B-players (Fig. S1). Depending on the treatment (see below), there are either six or one Cplayers. Active participants fill out a questionnaire which assessed their current and past 
medical history, use of medication (including contraception), food intake (including alcohol, caffeine, nicotine), type and duration of physical activity in the past 24 hours, length, and body weight, and gender and age.

We created three treatments. For all treatments, Stage I involved the real-effort cognitive task with the monetary payoff based on the individual's score (see below). The baseline-treatment group (B-T) only performed this task. In the other two treatments participants anticipated and performed Stage II in which they had to report to a peer seated in a separate office (Fig 1A). More specifically, in the 'Performance Ranking' treatment (PR-T), each participant individually and privately reports to the same peer and (truthfully) reads aloud his/her score as well as the ranking among the other participants in that treatment group. This allows the peer to compare performances. In the 'No-Ranking' treatment (NR-T), each participant reports to a different peer and (truthfully) reads aloud the score, but not the rank. This distinction uses the fact that ranking is by definition positional to isolate the mere effects of having to report one's result to a peer from the effects of performance ranking, i.e., being compared to others by a stranger (Schram et al., 2018). In both NR-T and PR-T, after finishing the instructions, each individual was taken to a C-player (each seated in a separate room) and reads aloud a text stating that $\mathrm{s} / \mathrm{he}$ will return after the task to report her/his performance (see Section 6 of the $S I$ ). This was done to increase the anticipation of having to later report to the C-player. At the end of Stage 2 each active participant reported (one at a time) to a different C-player and reads aloud the own score. In the NR-T, subjects knew their ranking but did not report this ranking to the C-player. In PR-T, each active participant reported, one at a time, to the same C-player and read aloud the own score and the rank amongst the active 
players from printed texts provided by the experimenter. In both treatments, C-players were not informed about the task, but were told that high scores indicate better performance than low scores. A-players know that the C-players do not know the task.

B-players are instructed about this cognitive task and perform the task without further interaction with other players. A-players are informed before the task that they will be required to report their performance to a C-player after completion. The instructions of both A- and B-players emphasize that this task has been shown to correlate positively with success in professional life.

\section{Cognitive Performance}

In stage 1 of the experiment, active participants are presented with a sequence of pairs of 10x10 matrices filled with two-digit numbers. These matrices appear at the bottom half of their computer monitor (Fig. S2). As described in Schram et al., 2018, for each pair of matrices each participant has to individually find the highest number in the left matrix and the highest number in the right matrix and to calculate the sum of these two numbers. This sum must be entered in the window at the center-top of the monitor (Fig. S2). A correct answer yields one euro. After a number has been entered, two new matrices appear, regardless of whether the sum was correct or not.

The task continues for 15 minutes. We apply this piece-rate remuneration in all of our treatments. Participants are informed that the numbers were 'randomly generated'. We did not draw from a uniform distribution, however, because this would have led with a high 
probability to very high sums. Instead, we first drew for each cell a random number between 40 and 99, say K. Subsequently, we drew a random number between 10 and $\mathrm{X}$ with equal probability.

\section{Hormonal Measurement}

Before the instructions of stage 1 are distributed among the active participants, we collected their saliva samples using standard procedures (Bosch et al., 2011; ; Granger et al., 2004; Riad-Fahmy et al., 1987). To prevent abnormalities in salivary measures, participants were asked not to smoke, eat or drink anything except water, and to not brush their teeth two hours prior to participation. Participants provided saliva samples before they received instructions for Stage 1 and then again directly after completing Stage 2. Subjects were handed a $25 \mathrm{ml}$ sterile polypropylene tube, asked to swallow all saliva in their mouths, and then allow saliva to collect for 3 minutes, spitting once a minute. The first (baseline) sample was taken after a 25-minute habituation period and the second sample was taken after the cognitive task had been completed. Collected samples were put on ice immediately and within an hour stored at -20 degrees Celsius. Upon completion of the study all samples were transported and analyzed for testosterone and cortisol at the University of Utrecht Medical Center.

\section{Results}

In initial analyses we examined the simple relationship between experimental treatment, cognitive performance, and hormonal adaptations in two planned contrasts: the first compared baseline (B-T) against the two reporting treatments (NR-T and PR-T combined) 
and the second compared performance ranking (PR-T) against the no-ranking treatments (NR-T and B-T combined). For cognitive performance, both contrasts were significant. Participants performed better when performance had to be reported rather than not, $t(\mathrm{xx})$ $=\mathrm{xxxx}, p=0.0 \mathrm{x}$, and they performed better when performance would be ranked rather than not, $t(\mathrm{xx})=\mathrm{x} . \mathrm{xxx}, p=0.045($ Fig $2 \mathrm{~A})$. For change in testosterone, we likewise find a stronger decline in testosterone when performance had to be reported rather than not, $t(\mathrm{xx})=\mathrm{xxxx}, p=0.0 \mathrm{x}$, and when performance would be ranked rather than not, $t(\mathrm{xx})=$ x.xxx, $p=0.045$ (Fig 2B). For changes in cortisol no significant effects were found, both $t<1$ (Fig 2C). Furthermore, decreasing testosterone (but not cortisol) positively relates to cognitive performance (Fig 2D).

To account for interdependencies between anticipating performance ranking, hormonal adaptations and cognitive performance, along with possible moderation by participant gender and lifestyle (see $S I$ ), we followed-up these initial analyses with a simultaneous equations model, formalized in eqs. (1):

$$
\left\{\begin{array}{c}
\Delta T=\alpha_{0}+\alpha_{1} G+\alpha_{2} X+\alpha_{3} X \cdot G+\alpha_{4} Y+\alpha_{5} Z+\varepsilon_{1} \\
\Delta C=\beta_{0}+\beta_{1} G+\beta_{2} X+\beta_{3} X \cdot G+\beta_{4} Y+\beta_{5} Z+\varepsilon_{2} \\
P=\gamma_{0}+\gamma_{1} G+\gamma_{2} \Delta T+\gamma_{3} \Delta T \cdot G+\gamma_{4} \Delta C+\gamma_{5} \Delta C \cdot G+\varepsilon_{3}
\end{array}\right.
$$

The first two equations of (1) depict the relationships in Fig. 1B and the third equation depicts the relationship in Fig 1C. $\Delta T(\Delta C)$ denotes the change in testosterone (cortisol) level; $G$ denotes gender (with $0=$ female; $1=$ male); $X$ denotes anticipated performance ranking (represented by three treatment groups: $B-T, N R-T$, and $P R-T$ ); $Y$ denotes initial hormone levels and $Z$ denotes lifestyle variables; $P$ denotes cognitive performance. The coefficients $\alpha_{i}, \beta_{i}, \gamma_{i}$ quantify the relationships indicated by arrows in Fig $1 \mathrm{BC}$ and will be 
estimated using our experimental data. Note that some of these constitute vectors of coefficients. For example, because anticipated performance ranking $(X)$ comprises a vector of two elements (the treatment dummies $N R-T$ and $P R-T$, with B-T serving as a benchmark), $\alpha_{2}$ and $\beta_{2}$ each constitute a vector of two coefficients. Our use of the interaction terms $(X \cdot G, \Delta T \cdot G, \Delta C \cdot G)$ allows the effects of anticipated performance ranking on hormonal change and the effects of hormonal change on performance to differ between men and women. Finally, the $\varepsilon_{i}, i=1,2,3$ denote random disturbance terms. We cannot assume these to be independently distributed because of the simultaneous equations structure where the dependent variables of the first two equations enter as independent variables in the third.

To account for the correlations between independent variables and disturbances that follow from these simultaneous equations, we apply a 3SLS regression framework (28). The coefficients $\alpha_{2}, \alpha_{3}, \beta_{2}, \beta_{3}, \gamma_{2}, \gamma_{3}, \gamma_{4}, \gamma_{5}$ capture the main relationships of interest. A first estimation of the coefficients showed that we cannot reject the null hypotheses that $\gamma_{3}=0$ and $\gamma_{5}=0$ (27). This means that there is no difference between men and women in how performance responds to hormonal adaptations. We therefore drop the interaction terms $\Delta T \cdot G, \Delta C \cdot G$ from the third equation in (1). Table 1 presents the coefficients estimated for the remaining relationships. Consistent with the results shown in Fig 2 we find that anticipating performance ranking (measured by the $\alpha_{2}$ and $\beta_{2}$ coefficients for $P R-T$ ) predicts a reduction in testosterone, and an increase in cortisol, and that reductions in testosterone and increases in cortisol enhance cognitive performance (as measured by $\gamma_{2}$ and $\gamma_{4}$ ). We observe no moderation of gender on the effects of anticipated performance ranking on changes in testosterone ( $\alpha_{3}$ for PR-T). For changes in cortisol, the effects are 
observed only for men (for women, $\beta_{2}+\beta_{3} \approx 0$ for PR-T). Finally, the increase in cortisol observed for NR-T and PR-T $\left(\beta_{2}\right)$ are of equal size and both significant. This means that for cortisol, but not for testosterone, simply reporting to a peer has the same effect as being ranked.

We concluded our analyses by examining the model fit. We did a 100 -fold repetition of outof-sample estimation. For every repetition we randomly selected an estimation group $E$ and a prediction group $P$. Every subject had a 50\% chance of being in either. In every repetition we estimated the model on $E$ and predicted the number of correct summations for each subject in $P$. In every repetition we then calculated for every subject in $P$ a variable $F$, which is the predicted number of correct summations divided by the observed number. If the prediction for an individual is precise, then $F=1$. A prediction that is $10 \%$ too high, for example, gives $F=1.1$. For every repetition, we calculated the average $F$ in $P$. We did so separately for each treatment (B-T, NR-T, PR-T). This gives 3 numbers per repetition and 300 in total. The observed values per treatment, ordered from low to high, show some overestimation (most values are larger than 1; Fig 3A), and that $82 \%$ of the predictions are between $-20 \%$ and $+20 \%$ of the observed number. Across all repetitions, the average prediction is very accurate (Fig 3B).

\section{Discussion and Conclusion}

Taken together, our results thus show that anticipating performance ranking causes a decrease of $18.37 \mathrm{pmol} / \mathrm{L}$ of testosterone and (for men) an increase of $1.90 \mathrm{nmol} / \mathrm{L}$ of cortisol, which accounts for approximately $\left(-0.10^{*}-18.37=\right) 1.8$ and $(1.90 * 0.47=) 0.9$ 
correct summations, respectively. Put differently, the hormonal adaptions to anticipated performance ranking associate with 2.7 (1.8) additional correct summations for men (women) which is almost 19\% (almost 14\%) of the mean number of correct summations that males (females) have when they perform the task in isolation. Thus, the anticipation of being ranked by a peer decreases testosterone and increases cortisol, and through this combination of reduced testosterone and increased cortisol cognitive performance can improve quite substantially. These findings are similar for males and females, but stronger for males, and remain whether or not we control for a range of life-style variables. Accordingly, we conclude that anticipating performance ranking enhances cognitive performance because such anticipation impacts hormonal adaptations in both testosterone and cortisol.

Others before us have observed effects of social comparison and performance ranking embedded in competitive incentive structures. Whereas competitive incentives can produce a surge of testosterone that in turn facilitates competitive performance, being observed has been associated with increased cortisol and, sometimes, reduced performance. Here we find a diametrically different pattern of results when competitive incentives are eliminated. Merely anticipating being ranked for performance reduces testosterone and increases cortisol, and increased cognitive performance. This suggests that the anticipation of performance ranking per se results in qualitatively different hormonal adaptations than does working under competitive incentives and while being observed by others. These effects are non-trivial as under continued exposure to specific external pressures, such as anticipated performance ranking, the associated hormonal adaptations of the kind 
observed here can exert structural effects on brain and behavior (Sapolsky, 2005, 2017). As noted, performance ranking is omnipresent and an almost unavoidable fact of both professional and recreational life. Our results suggest that this formal and informal institutionalization of performance ranking can impact human cognitive performance. Even in the absence of economic incentives, merely anticipating performance ranking already increases cognitive performance when and because it reduces overconfidence and motivates people to go the extra mile. 


\section{References}

Anderson S, Ertac S, Gneezy, U, List JA, Maximiano S. (2013). Gender, competitiveness, and socialization at a young age: Evidence from a matrilineal and a patriarchal society. Review of Economic Statisitics, 95, 1438 - 1443.

Bosch, JA, ECI Veerman, EJ de Geus, \& GB Proctor (2011). “A-Amylase as a Reliable and Convenient Measure of Sympathetic Activity: Don’t Start Salivating Just Yet!” Psychoneuroendocrinology, 36, 449-53.

Buckert, M., Schwieren, Ch. Kudielka, B., Fiebach, Ch. (2015). How stressful are economic competitions in the lab? Journal of Economic Psychology.

Buser T, Dreber A, Mollerstrom, J. (2017). The impact of stress on tournament entry. Experimental Economics, 20, 506-530.

Chen, Williamson \& Zhou. (2012). Reward system design and group creativity: An experimental investigation. Accounting Review, 87, 1885 - 191.

Cooke A, Kavussanu M, McIntyre D, Ring C. (2014). Effects of competition on endurance performance and the underlying psychological and physiological mechanisms. Biological Psychology, 86, 370 - 378.

De Dreu CKW, Nijstad BA. (2008). Conflict and creativity: Threat-rigidity or motivated focus? Journal of Personality and Social Psychology, 95, 648-661.

Dickerson SS \& Kemeny ME. (2004). Acute stressors and cortisol responses: A theoretical integration and synthesis of laboratory research. Psychological Bulletin, 130, 355-391.

Eisenegger C, Fehr E. (2011). The role of testosterone in social interaction. Trends in Cognitive Science, 15, $263-274$.

Eisenegger, C, M Naef, R Snozzi, M Heinrichs, and E Fehr. (2010). “Prejudice and Truth about the Effect of Testosterone on Human Bargaining Behaviour." Nature 463, 7279. 
Faul, F., Erdfelder, E., Lang, A.-G., \& Buchner, A. (2007). G*Power 3: A flexible statistical power analysis program for the social, behavioral, and biomedical sciences. Behavior Research Methods, 39, 175-191.

Festinger L. (1954). A theory of social comparison. Human Relations, 7, 117.

Gneezy U, Rustichini A. (2000). Pay enough or don't pay at all. Quarterly Journal of Economics, 115, 791-810.

Goldfarb EV, Froboese MI, Cools R \& Phelps E. (2016). Stress and cognitive flexibility: Cortisol increases are associated with enhanced updating but impaired switching. Journal of Cognitive Neuroscience, 29, 14 - 24.

Gouchie C, Kimura, D. (1991). The relationship between testosterone levels and cognitiveability patterns. Psychoneuroendocrinology, 16, 323 - 334.

Granger, D.A., Shirtcliff. E.A., Booth, A., Kivlighan, K.T, \& Schwartz, E.B. (2004). The 'Trouble' with Salivary Testosterone. Psychoneuroendocrinology, 29 1229-1240.

Harrison LK, Denning S, Easton HL, Hall JC, Burns VE, Ring C \& Carroll D. (2001). The effects of competition and competitiveness on cardiovascular activity. Psychophysiology, 38, 601-606.

Jost JT, Banaji MR, Nosek BA. (2004). A decade of system justification theory: Accumulated evidence of conscious and unconscious bolstering of the status quo. Political Psychology, 25, 881 - 919.

Kelsey RM, Blascovich J, Leitten CL, Schneider TR, Tomaka J \& Wiens S. (2000). Cardiovascular reactivity and adaptation to recurrent psychological stress: The moderating effects of evaluative observation. Psychophysiology, 37, 748 - 756. 
Mazur A, Booth, A. Testosterone and dominance in men (1998). Behavioral and Brain Sciences, 21, 353+.

Newman ML, Sellers, JG, Josephs, RA. (2005). Testosterone, cognition, and social status. Hormones and Behavior, 47, 205 - 211.

Oei NYL, Everaerd WTAM, Elzinga BM, Van Well S \& Bermond B. (2006). Stress, 9, 133 141.

Pashler H, Johnston JC, Ruthruff E. (2001). Attention and performance. Annual Review of Psychology, 52, 629-651.

Riad-Fahmy D, Read GF, Walker RF, Walker SM, Griffiths K. (1987). Determination of ovarian steroid hormone levels in saliva. An overview. Journal of Reproductive Medicine, 32, 254-72.

Sapolsky RM (2005). The influence of social hierarchy of primate health. Science, 308, 648 652.

Sapolsky RM. (2017). Behave: Humans at their best and worst. New York, Penguin.

Schmader T, Johns M \& Forbes C. (2008). An integrated process model of stereotype threat effects on performance. Psychological Review, 115, 336 - 356.

Schram, A.J.H.C., J. Brandts and K. Gërxhani. Experimental Economics (2018).

Staw BM, Sandelands, LE, Dutton JE. (1981). Threat-rigidity effects in organizational behavior-A multi-level analysis. Administrative Science Quarterly, 26, 501-524.

Taylor SE, Lobel M. (1989). Social-comparison activity under threat - Downward evaluation and upward contacts. Psychological Review, 96, 569 - 575.

Twenge JM, Gentile B, DeWall CN, Ma D, Lacefield K, Schurtz DR. (2009) Birth cohort increases in psychopathology among young Americans, 1938-2007. A cross-temporal meta-analysis of the MMPI. Clinical Psychology Review, 30, 145 - 154. 
Weber, M., \& Schram, A.J.H.C. (2016). The non-equivalence of labour market taxes: A realeffort experiment. Economic Journal, 127, 2187-2215.

\section{Author Acknowledment and Contribution}

This work was supported by the Behavioral Economics Research Priority Grant to AS and a Spinoza Award from the Netherlands Science Foundation to CKWDD (NWO SPI-57-242). Author Contributions: CKWDD, KG and AS designed the study, discussed results, and wrote the paper. AS and KG implemented the study and analyzed the data. We thank Michael Giffin and Eliska Prochazkova for their assistance with data collection. The authors declare no conflict of interest. 
Table 1 Coefficients of model (1) were estimated with 3SLS (N=95). The full regression model includes a gender dummy $(0=$ male; $1=$ female $)$, lifestyle variables, and benchmark levels of testosterone and cortisol. A full overview of the estimates is in (Supplementary Materials, 27). Results for NR-T and PR-T are relative to the benchmark of conducting the task without reporting performance (B-T). $*(* * / * * *)$ indicates that the coefficient concerned is statistically significantly at the 10\%-(5\%-/1\%-) level (Wald tests).

\begin{tabular}{|c|c|c|c|}
\hline Relationship in Fig. 1 & Coefficient in Eq. (1) & Estimated coefficient & Standard error \\
\hline \multirow{2}{*}{$\begin{array}{l}\text { Anticipated performance ranking } \\
\text { affects change in testosterone }\end{array}$} & $\alpha_{2}(\mathrm{NR}-\mathrm{T})$ & -0.69 & 9.16 \\
\hline & $\alpha_{2}(\mathrm{PR}-\mathrm{T})$ & -18.37 & $8.71^{* *}$ \\
\hline \multirow{2}{*}{$\begin{array}{l}\text { Moderation of being female on } \\
\text { performance ranking effect on } \\
\text { testosterone }\end{array}$} & $\alpha_{3}(\mathrm{NR}-\mathrm{T})$ & -15.26 & 15.60 \\
\hline & $\alpha_{3}(\mathrm{PR}-\mathrm{T})$ & 10.10 & 15.83 \\
\hline \multirow{2}{*}{$\begin{array}{l}\text { Anticipated performance ranking } \\
\text { affects change in cortisol }\end{array}$} & $\beta_{2}(\mathrm{NR}-\mathrm{T})$ & 2.96 & $1.00^{* * *}$ \\
\hline & $\beta_{2}(\mathrm{PR}-\mathrm{T})$ & 1.90 & $0.95^{* *}$ \\
\hline \multirow{2}{*}{$\begin{array}{l}\text { Moderation of being female on } \\
\text { performance ranking effect on cortisol }\end{array}$} & $\beta_{3}(\mathrm{NR}-\mathrm{T})$ & -5.12 & $1.71^{* * *}$ \\
\hline & $\beta_{3}(\mathrm{PR}-\mathrm{T})$ & -2.03 & 0.24 \\
\hline $\begin{array}{l}\text { Effect of change in testosterone on } \\
\text { performance }\end{array}$ & $\gamma_{2}$ & -0.10 & $0.04^{* *}$ \\
\hline $\begin{array}{l}\text { Effect of change in cortisol on } \\
\text { performance }\end{array}$ & $\gamma_{4}$ & 0.47 & $0.27^{*}$ \\
\hline
\end{tabular}


Fig. 1 Overview of the Experiment and Model Summary. (A). Timeline of the experimental procedures and measurements. (B/C) Hypothesized relations between anticipated performance ranking and cognitive performance through hormonal adaptations. Symbols represent the relationships defined in eqs (1). (B) Anticipated performance ranking predicts changes in testosterone and cortisol, controlling for gender and lifestyle (27). (C) Changes in testosterone and cortisol predict cognitive performance, possibly moderated by participant gender.

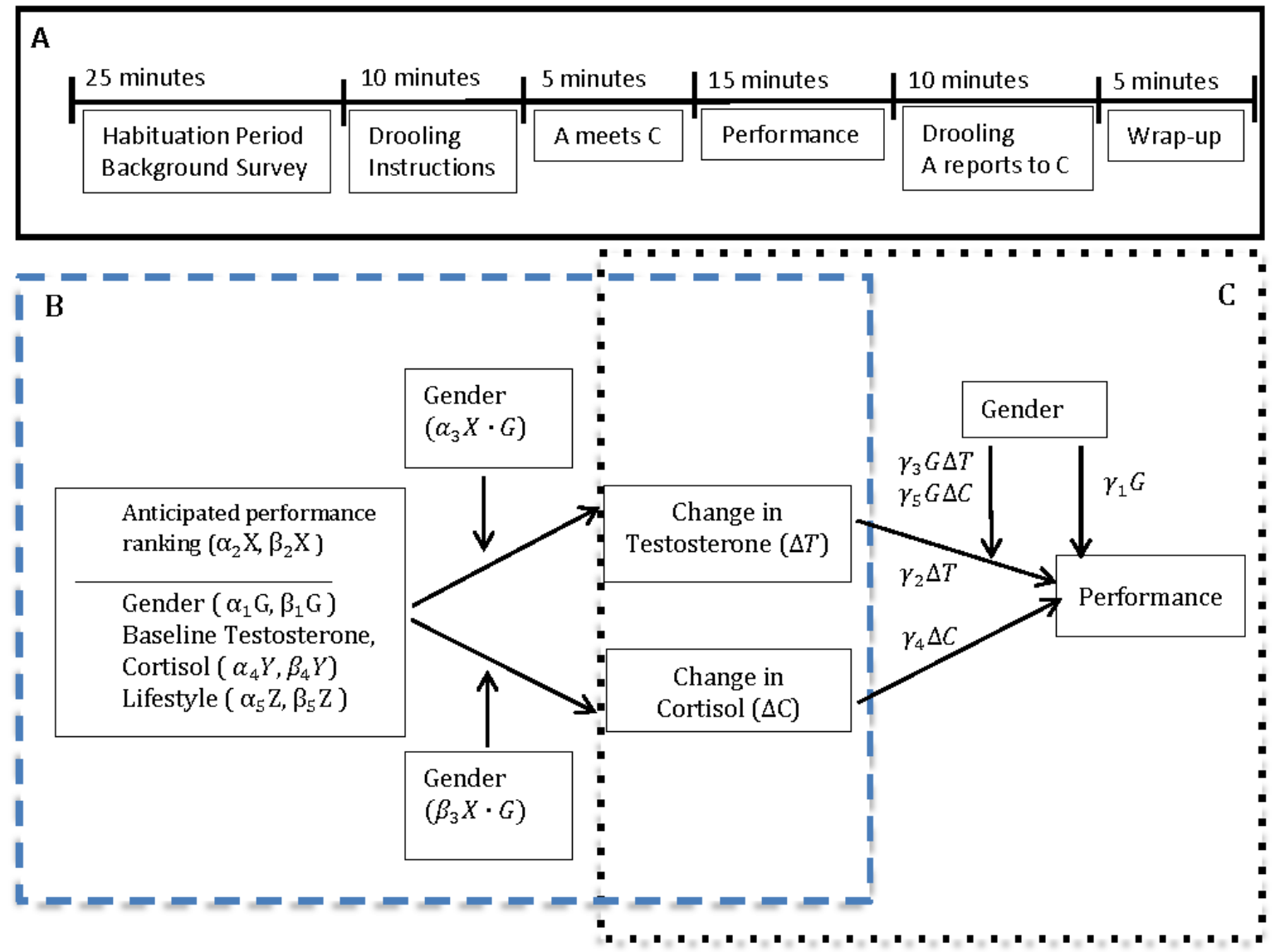


Fig. 2 Responses to Anticipated Performance Ranking. (A) Cognitive performance (number of correct responses) as a function of treatment (shown Mean \pm SE). (B) Changes in testosterone $(\mathrm{pmol} / \mathrm{L})$ from pre- to post-task (shown Mean \pm SE). (C) Changes in cortisol $(n \mathrm{~mol} / \mathrm{L})$ from pre- to post-task (shown Mean \pm SE). (D) Correlations between change in testosterone (shown on left $y$ axis) and cortison (shown on right y-axis) and cognitive performance. Green diamonds (red triangles) show individual pairings of cognitive performance and change in testosterone (cortisol). Green (red dashed) line shows best fitting linear regression of change in testosterone (cortisol) to cognitive performance, with $r=-0.22, p=0.029$. and $r=-0.08, p=0.414$, respectively.

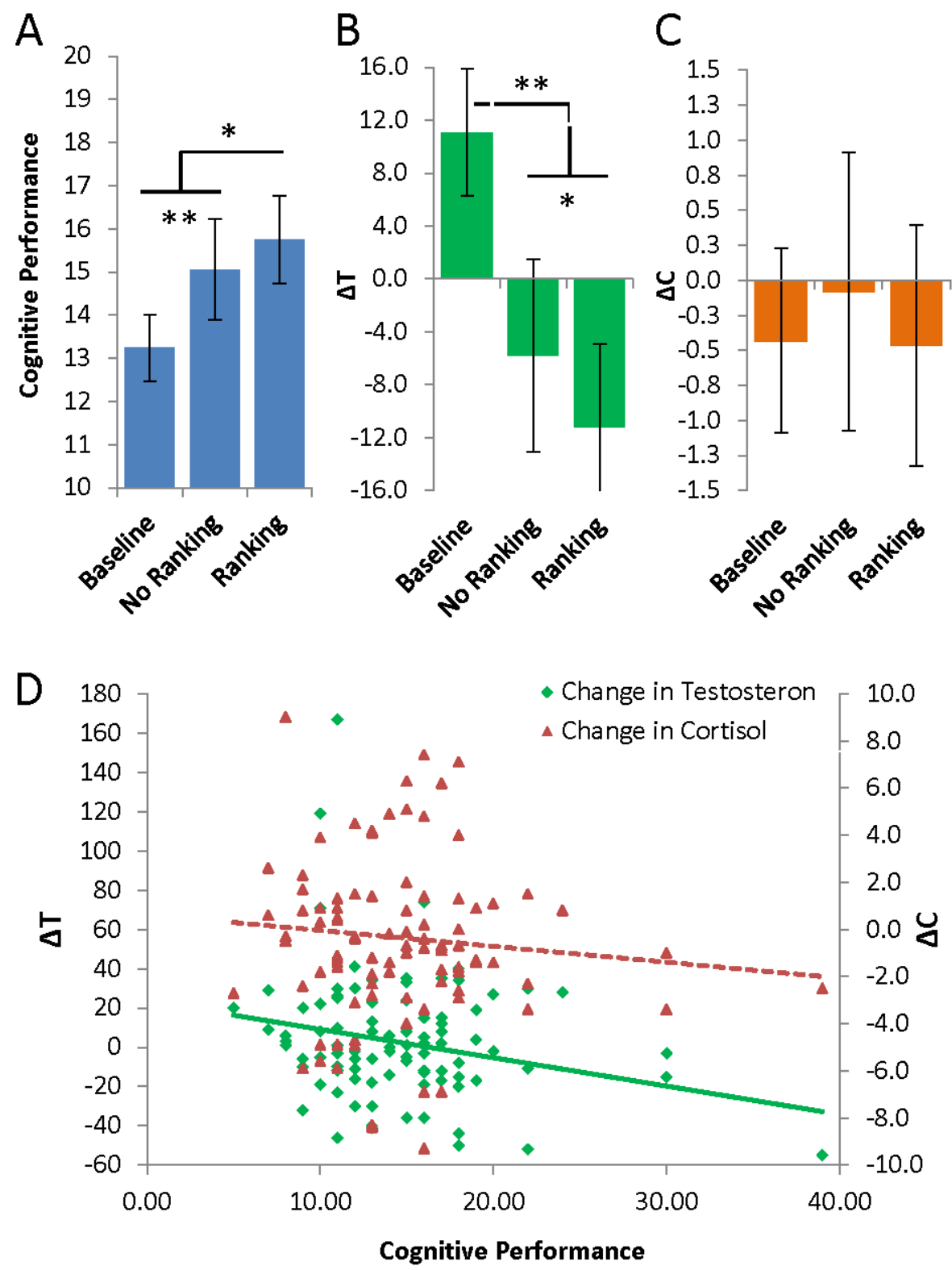


Fig 3 Predicted and Observed Performance. (A) Observed values per treatment, ordered from low to high. Perfect prediction is achieved when $F=1.0$. Observed cases within the dashed area are predicted with 80\% accuracy. (B). Out-of-sample predictions, showing the average performance for 100 subsamples of $50 \%$ of the data as estimated by the model (light blue) on the remaining $50 \%$ compared to their observed performance (dark blue).
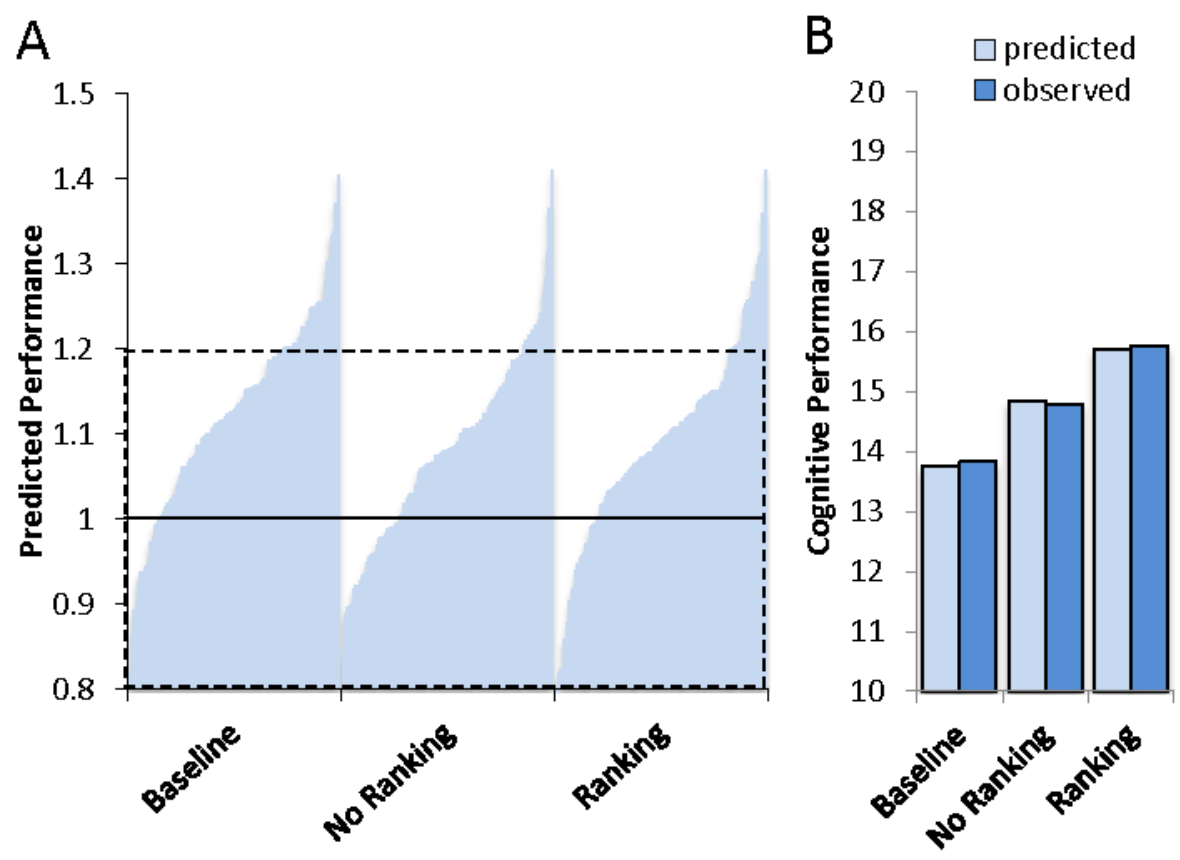